\title{
GLOBAL STRUCTURE OF ORDINARY DIFFERENTIAL EQUATIONS IN THE PLANE
}

\author{
BY \\ L. MARKUS( ${ }^{(1)}$
}

\section{Statement of PRoblems AND RESUlts}

1. Introduction. In this paper we shall develop a topological analysis and classification of real, first order, ordinary differential equations defined in the Euclidean plane.

Definition. A differential system $\mathcal{S}$ shall mean $\left({ }^{2}\right) \dot{x}=f(x, y), \dot{y}=g(x, y)$ where $f(x, y)$ and $g(x, y) \in C^{(1)}$ (have continuous first partial derivatives in the Cartesian coordinates $(x, y))$ in a plane region $R$ (open, connected set). Furthermore the critical points (where $f=g=0$ ), each of which is a solution curve, are isolated.

In the traditional functional description of differential systems, for instance $y^{\prime}=-x / y$ with variables separable and $y^{\prime}=\left(y^{2}+x\left(x^{2}+y^{2}\right)^{1 / 2}\right) /(x y$ $\left.-y\left(x^{2}+y^{2}\right)^{1 / 2}\right)$ which is homogeneous, the classification is based on the superficial form of the functions. This description is not invariant under even the Euclidean group and is determined by the calculational trick by which the solutions are found explicitly. However, the solution curve families of the above two examples are qualitatively of the same form. Each family consists of simple closed solution curves about a unique critical point. Under a topological classification the two examples are equivalent.

Definition. Two differential systems (in fact, any two collections of curves) $S_{1}$ and $S_{2}$ defined in plane regions $R_{1}$ and $R_{2}$ respectively are $o$-equivalent in case there exists an $o$-homeomorphism (orientation preserving homeomorphism) of $R_{1}$ onto $R_{2}$ which induces a biunique mapping of the set of curves of $S_{1}$ onto the curves of $S_{2}$.

We consider only maps of solution curves as point sets and not maps of trajectories or "time" parametrized curves which trace out the solution curves. However if $S_{1}$ and $S_{2}$ are $o$-equivalent, one can introduce a new "time" along each solution of $S_{1}$, that is, multiply the functions of $S_{1}$ by a nonvanishing, continuous factor, so that the trajectories as well as the solutions of $S_{1}$ and $S_{2}$ correspond.

Presented to the Society, April 26, 1952; received by the editors March 21, 1952 and, in revised form, November 24, 1952.

(1) The author wishes to thank Professor G. Birkhoff for aid in preparation of his doctoral dissertation, of which this is a modification, for Harvard University. Most of the work was done under Project XXII of Contract N5ori-76 and Contract N5ori-07634 with the Office of Naval Research. family.

(2) $\dot{y}=d y / d t, y^{\prime}=d y / d x$. We use $\mathcal{S}$ for either the differential system or its solution curve 
The assumption that the critical points of a differential system are isolated and the attention paid to $o$-homeomorphisms rather than all homeomorphisms are introduced for convenience of statement and are not essential to the following theories.

We assume familiarity with only the basic qualitative theory of differential systems in the plane [11, Chap. 5] $\left(^{3}\right)$, however most of our results are valid, after minor changes, for orientable regular curve families filling any plane region, see Whitney [20] and Kaplan [8]. Our emphasis and results differ considerably from these latter purely topological works, since our primary interest lies in a structure theory which illuminates and unifies the classical analytical theory of differential equations.

2. Summary of results. We consider the geometry of global differential systems under the group of topological maps of the Euclidean plane $E$ onto itself. We find canonical representatives for the differential systems of the various $o$-equivalence classes and we order these by means of a complete set of invariants. Incidentally, our technique of classification illuminates the structure of every differential system and aids in the extension of certain local properties of differential systems to global theories.

As examples of these global theories we construct global, differentiable, integral functions for a wide class of differential systems. Also we investigate global Lie transformation groups and their invariants. Finally we correlate the topological classification of differential systems with the customary analytical description of the standard elementary types.

The theory of invariants of differential systems under certain subgroups of the group of local homeomorphisms has had a traditional evolution, see $[5 ; 13 ; 12 ; 3 ; 2]$. However, such a theory for global differential systems has had, as yet, very little development.

\section{SEPARATRIX AND CANONICAL REgION STRUCTURE ILLUSTRATED BY ELEMENTARY TYPES OF DIFFERENTIAL SYSTEMS}

3. Definitions of separatrices and canonical regions. Locally each solution curve of a differential system $S: y^{\prime}=f(x, y)$ (or $\dot{x}=1, \dot{y}=f(x, y)$ ) is topologically an open line interval. Moreover the collection of solutions are $o$-equivalent to parallel straight lines. The $o$-homeomorphism is defined easily by using the orthogonal trajectories, each of which intersects each solution of $S$ in precisely one point. Also there is always an integral $\psi(x, y) \in C^{(1)}$, defined locally, which is constant on each solution curve of $\mathcal{S}$ but which is not constant on any open set.

These basic local properties are not valid for global differential systems. In particular, the lack of global transversals, such as orthogonal trajectories, which intersect every solution curve, is the most serious difficulty.

(3) Numbers in brackets refer to the bibliography at the end of the paper. 
We shall decompose each global solution family into subsets, to be called canonical regions, in each of which the local theories of differential systems are true. The solution curves which bound the canonical regions will be distinguished topologically and will be called separatrices. It is by a combinatorial analysis of the canonical regions and separatrices that our classification is effected.

For preciseness we recall: a curve is a continuous image of the real line; an (open, half-open, compact) arc is a homeomorphic image of a (corresponding) line interval; a plane region $R$ is filled by a collection $S$ of curves in case each curve of $S$ lies in $R$ and each point of $R$ is contained in exactly one curve of $S$; if $S$ is the solution family of a differential system which fills the plane $E$, then $S$ also fills a subregion $R \subset E$, that is, $R$ is the union of curves of $S$ if and only if the boundary of $R$ in $E$ is the union of curves of $S$.

Definition. A collection $S$ of curves filling a plane region $R$ is parallel in case $S$ in $R$ is $o$-equivalent to either the plane filled by parallel lines, or the punctured plane filled by concentric circles, or the punctured plane filled by rays from the deleted point.

For abbreviation we say that $R$ is parallel and refer to the three cases as strip, annular, and radial or spiral, respectively. A strip region is simplyconnected whereas the other two types are doubly-connected. But the annular region is topologically distinguished in that it is filled by compact curves.

If $\mathcal{S}$ is parallel in $R$, then $\mathcal{S}$ admits a transversal (an $\operatorname{arc} \Gamma \subset R$ which intersects each curve of $\mathcal{S}$ in exactly one point) which is an open arc for strip and annular regions but a half-open arc for spiral regions. However, if $\mathcal{S}$ in $R$ admits a transversal, then $R$ is not necessarily parallel except under further hypotheses to be discussed later.

The separatrices (which properly include all the separatrices of Lefschetz $[11$, p. 170] and the bounding cycles of Kaplan [8, p. 177]) are solutions which form barriers to the extension of transversals. The separatrices are solutions which differ from their neighbors either in their limit sets, $S_{+}$ $\equiv$ closure $[S(t \geqq 0)]-S(t \geqq 0)$ or $S_{-} \equiv$ closure $[S(t \leqq 0)]-S(t \leqq 0)$, or in their behavior in the large.

Definition. A solution curve $S$ of a differential system $\mathcal{S}$, defined in the entire $\left(^{4}\right)$ plane $E$, is a separatrix in case $S$ is not embedded in a parallel neighborhood $N$ such that (1) every solution of $\mathcal{S}$ in $N$ has the same limit sets $S_{+}$and $S_{-}$, and (2) $N$ is bounded by $S_{+} \cup S_{-}$and exactly two solutions $S^{\prime}$ and $S^{\prime \prime}$ for which $S_{+}^{\prime}=S_{+}^{\prime \prime}=S_{+}$and $S_{-}^{\prime}=S_{-}^{\prime \prime}=S_{-}$.

We note that the three sets $S^{\prime}, S^{\prime \prime}$, and $S_{+} \cup S_{-}$are always disjoint and furthermore $N$ can always be taken to be either strip or annular.

The following special cases of separatrices are immediate consequences of the definition. Every critical point is a separatrix. A closed orbit $S$ (simple

$\left.{ }^{4}\right)$ If $S$ fills a simply-connected plane region $R$, then the corresponding definitions are made using the relative topology in $R$. Usually we take $R=E$. 
closed solution curve) is a separatrix if and only if every neighborhood of $S$ intersects a line homeomorph solution which is clearly a spiral. If the limit sets $S_{+}$and $S_{-}$of a solution curve $S$ each contain more than one point, then $S$ is not a separatrix.

If a solution curve $S$ has arbitrarily close neighbors $S^{\prime}$ such that either $S_{+}^{\prime} \neq S_{+}$or $S_{-}^{\prime} \neq S_{-}$, then $S$ is a separatrix. However it is possible for a solution $S$ to be a separatrix even though all its neighbors have the same limit sets. In fact, there exist differential systems which have no critical points in the plane, and thus $S_{+} \cup S_{-}$is empty for each solution curve $S$, and yet such that every solution curve is a separatrix. However for most of the commonly encountered differential systems, we shall see that the separatrices are denumerable.

Definition. Let $S_{1}$ and $S_{2}$ be solution curves of a differential system $\mathcal{S}$ filling $E$. We say $S_{1}$ is parallel to $S_{2}$ in case both $S_{1}$ and $S_{2}$ are contained in one parallel region $N$ such that (1) every solution $S \subset N$ has the same positive and the same negative limit sets, and (2) $N$ is bounded by $S_{+} \cup S_{-}$together with exactly two distinct solutions $S^{\prime}$ and $S^{\prime \prime}$ for which $S_{+}^{\prime}=S_{+}^{\prime \prime}=S_{+}$ and $S_{-}^{\prime}=S_{-}^{\prime \prime}=S_{-}$.

The parallel relation is symmetric and an investigation [11, p. 172] of the nature of the limit sets $S_{+}$and $S_{-}$shows that it is also transitive, but it is not always reflexive. A characteristic property of a separatrix $S$ is that $S$ is parallel to no solution curve of $\delta$.

If $\delta$ has no critical points in $E$, another definition of the separatrix curves can be established by means of a topology on the collection of solution curves. Here every solution of $\mathcal{S}$ separates $E$ into two disjoint regions and each pair $S_{1}$ and $S_{2}$ of solutions bounds a unique region. We designate the sets of curves of $\delta$ filling such regions bounded by pairs of curves of $\delta$ as an open neighborhood basis for $\delta$. Then the separatrices of the noncritical differential system $\mathcal{S}$ are precisely those elements of $\mathcal{S}$ which are not closed in this topology. Each equivalence class of $\mathcal{S}$, under the parallel relation, can be seen to be a onedimensional manifold and, indeed, to fill one entire canonical region.

THEOREM 3.1. Let $\subseteq$ be the union of all separatrices of a differential system $S$ fitling $E$. Then $\subseteq$ is closed in $E$.

Proof. Each non-separatrix is embedded in an open plane neighborhood filled by non-separatrices. Thus the complement of $\subseteq$ is open in E. Q.E.D.

Definition. Each component of the complement of $\mathfrak{S}$, the union of all separatrices of $\mathcal{S}$ filling $E$, is called a canonical region of $\mathcal{S}$.

REMARK. Let $S^{\prime}$ and $S^{\prime \prime}$ be solutions of $S$ and lie in one canonical region of $\delta$. Then $S_{+}^{\prime}=S_{+}^{\prime \prime}$ and $S_{-}^{\prime}=S_{-}^{\prime \prime}$. Also $S^{\prime}$ is a closed orbit if and only if $S^{\prime \prime}$ is a closed orbit.

It is easy to construct differential systems having an infinite number of separatrices. For example, $\dot{x}=(2 / \pi)\left(1 /\left(y^{2}+1\right)\right)\left(1+\sin ^{2} x\right), \dot{y}=-(4 / \pi) \tan ^{-1} y$ 
- $\sin x \cos x$ has a canonical region bounded by a countable set of separatrices which are the components of the locus $(2 / \pi) \tan ^{-1} y\left(1+\sin ^{2} x\right)= \pm 1$. However, for the theory developed below, it is important that although there may be an infinitude of separatrices, they should not cluster about some solution curve which is then a limit separatrix.

Definition Let $\subseteq$ be the union of all separatrices of a differential system $S$ filling $E$. If a separatrix $S \subset$ closure [ $\subseteq-S$ ], then $S$ is called a limit separatrix.

REMARK. A critical point is not a limit separatrix if and only if it is a boundary point of just one canonical region which must then be annular or spiral. A closed orbit separatrix which is not a limit separatrix belongs to the boundary of two canonical regions neither of which is strip parallel but one of which is spiral parallel filled by solutions which approach the given closed orbit. If $S$ is noncritical, a separatrix $S$ bounds two canonical regions if and only if $S$ is not a limit separatrix; a limit separatrix bounds one or no canonical regions.

4. Examples of separatrices and canonical regions of "elementary" differential systems. We shall state without proofs, see [14] and [15], the separatrix structure of several nontrivial examples.

CASE 1. Let $S$ be $y^{\prime}=g(x, y) / f(x, y)=h(x, y) \in C^{(1)}$ in $E$ or $\dot{x}=f(x, y)$, $\dot{y}=g(x, y)$ with $\left(^{5}\right) f(x, y) \neq 0$. If $|h(x, y)|<K$ for some constant $K$, then $S$ is $o$-equivalent to $y^{\prime}=0$ in $E$. Thus $S$ has no separatrices and one strip canonical region. In fact, if either $h(x, y)<K(x)$ or $h(x, y)>K(x)$ for some continuous function $K(x),-\infty<x<\infty$, then $\delta$ is $o$-equivalent to $y^{\prime}=0$ in $E$.

CASE 2. Let $S$ be $y^{\prime}=h(x, y)$ with $h(x, y) \in C^{(1)}$ in $E$. Suppose for each number $x_{0}$ there exists an $\epsilon>0$ and a positive continuous function $\Omega(u)$ defined for $u>0$ such that:

$$
\left|h\left(x, y_{1}\right)-h\left(x, y_{2}\right)\right| \leqq \Omega\left(\left|y_{1}-y_{2}\right|\right)
$$

for all $\left|x-x_{0}\right|<\epsilon$ and $y_{1} \neq y_{2}$;

$$
\int_{1}^{\infty} \frac{d u}{\Omega(u)}=\infty, \quad \text { and also } \liminf _{u=\infty} \Omega(u)>0 .
$$

Then $S$ is $o$-equivalent to $y^{\prime}=0$ in $E$.

This example includes the classical Lipschitz case where $\Omega(u)=K u$ where $K>0$ is a constant depending on $x_{0}$, see $[6$, p. 20]. In particular if $|\partial h / \partial y|$ $<K(x)$, a continuous function on $-\infty<x<\infty$, then $S$ is $o$-equivalent to $y^{\prime}=0$ in $E$.

CASE 3. Every linear differential equation $y^{\prime}=a(x) y+b(x)$, where $a(x)$ and $b(x)$ are continuous, is $o$-equivalent to $y^{\prime}=0$ in $E$.

${ }^{(5)} f(x, y) \neq 0$ means $f(x, y)$ is nowhere zero. $f(x, y) \not 0$ means there is a point at which $f(x, y)$ is not zero. 
CASE 4. Let $S$ be $y^{\prime}=h(y)$ with $h(y) \in C^{(1)}$ in $E$. Then $S$ is $o$-equivalent to $y^{\prime}=0$.

CASE 5. Let $S$ be $\dot{x}=f(x, y), \dot{y}=g(x, y)$ with $f^{2}+g^{2}>0$ and $f(x, y), g(x, y)$ $\in C^{(1)}$ in $E$. Let $N(\rho)$ be the number of distinct points on the circle $x^{2}+y^{2}=\rho^{2}$ at which $\dot{r} \equiv(x f+y g) /\left(x^{2}+y^{2}\right)^{1 / 2}$ vanishes. If $\lim \inf _{\rho=\infty} N(\rho)<4$, then $S$ is $o$-equivalent to $y^{\prime}=0$. Thus $\dot{x}=\left(x^{2}+y^{2}+1\right) e^{y}, \dot{y}=x^{3} y$ is accounted for under this case but not under the preceding ones.

We shall see that every differential system having no separatrices is $o$-equivalent to $y^{\prime}=0$ in $E$, and this even under a $C^{(1)}$-homeomorphism. However, for differential systems with separatrices, topological equivalence does not imply equivalence under a differentiable change of coordinates.

There are no noncritical differential systems having just one separatrix. But there is precisely one $o$-equivalence class consisting of noncritical differential systems having just two separatrices and thus three strip canonical regions. This last-mentioned type has a special physical significance as described next.

CAsE 6. Let the dynamical system $\ddot{x}-f(x, \dot{x})=0$ be represented in the phase plane $(x, y=\dot{x})$ by $S: \dot{x}=y, \dot{y}=f(x, y)$ where $f(x, y) \in C^{(1)}$ in $E$. Suppose there are no stable phases, that is, $f(x, 0) \neq 0$. Then either there are no separatrices and $S$ is $o$-equivalent to $y^{\prime}=0$, or there are exactly two separatrices and $S$ is $o$-equivalent to $\dot{x}=y, \dot{y}=e^{x}$.

As an example of a dynamical system having no separatrices consider a particle moving vertically in a constant (nonzero) force field. For the other type of dynamical system consider a particle subjected to the inverse-square gravitational attraction of the earth. However, to comply with the hypothesis of no stable phases, we replace the gravitational field by a constant field below the surface of the earth. The two separatrices correspond to the "escape trajectory" and the trajectory of "free fall from infinity."

CASE 7. Let $\left\{a_{i}\right\}, i=0, \pm 1, \pm 2, \cdots$, be a finite or countable discrete set of real numbers with $a_{i}<a_{j}$ if $i<j$. Let $S$ be $\dot{x}=f(x, y), \dot{y}=g(x, y)$ with $f(x, y), g(x, y) \in C^{(1)}$, and $f^{2}+g^{2}>0$ in $E$, and $f(x, y)=0$ if and only if $x=a_{i}$. For each compact interval $\alpha \leqq x \leqq \beta$ containing none of the $\left\{a_{i}\right\}$, let $L(y ; \alpha, \beta)$ $=\max _{\alpha \leqq x \leqq \beta,|\eta|=y}|g(x, \eta) / f(x, \eta)|$. Suppose $\int_{u}^{\infty} d y / L(y ; \alpha, \beta)=\infty$ for each $u \geqq 0$. Then there are no limit separatrices of $\mathcal{S}$. Each canonical region is strip parallel and is bounded by at most four separatrices.

If the set $\left\{a_{i}\right\}$ is empty, then $S$ has no separatrices and is $o$-equivalent to $y^{\prime}=0$ in $E$.

We mention the special case of variables separable as in $\delta: \dot{x}=f(x)$, $\dot{y}=g(y)$ where $g(y) \neq 0$, and $\int_{0}^{\infty} d y / \max \{|g(y)|,|g(-y)|\}=\infty$, and the zeros of $f(x)$ are discrete.

Of special interest are the examples $\dot{x}=\sin x, \dot{y}=\cos x$ and $\dot{x}=\sin ^{2} x$, $\dot{y}=\cos x$ which have the same separatrices, the lines $x=0, \pm \pi, \pm 2 \pi, \cdots$, but which are not $o$-equivalent. 
CASE 8. Let $S$ be $\dot{x}=P(x, y) \exp P_{1}(x, y), \dot{y}=Q(x, y) \exp Q_{1}(x, y)$ where $P, P_{1}, Q, Q_{1}$ are polynomials of degree $n$, at most, and $P^{2}+Q^{2}>0$ in $E$. Then there are no limit separatrices; each canonical region is strip parallel and is bounded by at most $K(n)=24 n^{2}+62 n+39$ separatrices.

In the case where $P_{1}$ and $Q_{1}$ are constants we can take $K(n)=2 n+2$. Riccati and Bernoulli polynomial equations such as $y^{\prime}=\sum_{i=0}^{n} P_{i}(x) y^{n}$, where the $P_{i}$ are polynomials, are of this type.

CASE 9. Let $S$ be $\dot{x}=f(x, y), \dot{y}=g(x, y)$ with $f(x, y), g(x, y) \in C^{(1)}$, and $f^{2}+g^{2}>0$ in $E$, and let $R$ be a bounded plane region. Then there exists a simply-connected region $U$ such that $\bar{R} \subset U$ and $\mathcal{S}$, considered as a differential system in $U$, has only a finite number of separatrices. Thus $S$ in $U$ has no limit separatrices.

This result, together with Theorem 10.1 below, generalizes Kamke's theorem [7, p. 56].

CASE 10. Let $\delta$ be the homogeneous differential system $\dot{x}=f(x, y)$, $\dot{y}=g(x, y)$ where $g / f$ or $f / g$ is constant along each open ray leading from the origin, which is the unique critical point. There are a finite number of separatrices of $\mathcal{S}$, the origin and a finite number of rays from the origin. If there is only one separatrix, then there is just one canonical region which is then either annular or spiral. If there is more than one separatrix, then each canonical region is of strip type.

For the linear homogeneous system $\dot{x}=a x+b y, \dot{y}=c x+d y$ where the determinant $a d-b c \neq 0$, we distinguish three subcases. If $a d-b c<0$, this is a saddle point. Here the critical point and the four exceptional rays are separatrices; and there are four strip canonical regions. If $a d-b c>0$ and $a+d=0$, this is a center point with the critical point as unique separatrix and with one annular canonical region. If $a d-b c>0$ and $a+d \neq 0$, this is a spiral or else a nodal point with the critical point as unique separatrix and with one spiral canonical region.

CASE 11. Let $S$ be Lienard's equation [11, p. 188] or the special case of van der Pol's equation $\dot{x}=y+\mu\left(x-x^{3} / 3\right), \dot{y}=x$, with $\mu>0$. Here there are two separatrices, the critical point origin and the unique closed orbit. There are two spiral canonical regions.

\section{Structure of Differential Systems}

5. Parallel nature of canonical regions. In this section we shall demonstrate that each canonical region admits a transversal, is a parallel region, and consists of precisely one equivalence class of solutions under the parallel relation.

Suppose, at first, that $S$ fills a simply-connected region $R$ and therein $S$ has no critical points. Then each curve of $S$ divides $R$ into two regions. For each triple of distinct solutions $S_{1}, S_{2}, S_{3}$ of $\mathcal{S}$ either one of them, say $S_{2}$, separates the others and we write $S_{1}\left|S_{2}\right| S_{3}$, or else they form a cyclic triple, 
say counterclockwise in the order $S_{1}, S_{2}, S_{3}$, and we write $\left|S_{1} S_{2} S_{3}\right|^{+}$, see [8, p. 165].

LEMma 5.1. Let a differential system $S$ fill a simply-connected region $R$ and therein let $\mathcal{S}$ be noncritical. Then the following three statements are equivalent:

1. S contains no cyclic triples of distinct solutions,

2. $S$ admits an open transversal $\Gamma$ in $R$ which is a nonsingular, analytic arc nowhere tangent to a solution of $S$,

3. S is o-equivalent to $y^{\prime}=0$ in $E$.

Proof. See Theorems 1, 2, 3 in [15]. Q.E.D.

LEMma 5.2. Let a differential system $S$ fill a simply-connected region $R$ and therein let $S$ be noncritical. Then $S$ contains a separatrix in $R$ if and only if $S$ contains a cyclic triple of distinct solutions in $R$.

Proof. Suppose $\delta$ contained a separatrix but contained no cyclic triple of solutions. Then by Lemma $5.1, S$ in $R$ is $o$-equivalent to $y^{\prime}=0$ in $E$ which contains no separatrices. Since separatrices are defined as topological invariants, this is a contradiction and $S$ must thus contain a cyclic triple of solutions.

Conversely suppose $\delta$ contained a cyclic triple of solutions $S_{1}, S_{2}, S_{3}$ but $\delta$ contained no separatrix. Join $S_{1}$ to $S_{2}$ by a curve $\pi(t), 0 \leqq t \leqq 1$, which lies entirely within the region bounded by $S_{1}$ and $S_{2}$, except for the end points $\pi(0) \in S_{1}$ and $\pi(1) \in S_{2}$. Also construct $\pi(t)$ so that it does not intersect $S_{3}$.

Let $T$ be the set of all real numbers $0 \leqq t \leqq 1$ such that $t_{0} \in T$ in case the solution $S_{t_{0}}$, containing $\pi\left(t_{0}\right)$, lies within a strip region containing $S_{1}$ and bounded in $R$ by two curves of $S$. Now $T$ is open in $[0,1]$ but also closed since each solution can be embedded in a strip neighborhood which is bounded in $R$ by two curves of $S$. Thus $T=[0,1]$ and thus $S_{2}$ and $S_{1}$, together with all the solutions of $S$ which lie between them, lie in a strip region $N$. But $N$ contains the cyclic triple of solutions $S_{1}, S_{2}, S_{3}$ and this contradicts Lemma 5.1. Q.E.D.

THEOREM 5.1. Let $S$ be a differential system in a simply-connected region $K$. Then $S$ is o-equivalent to $y^{\prime}=0$ if and only if $S$ contains no separatrices.

Proof. If $S$ is $o$-equivalent to $y^{\prime}=0$, then $S$ clearly contains no separatrices. If $S$ contains no separatrices, then $S$ has no critical points in $R$. Then the two lemmas yield the conclusion that $S$ in $R$ is strip parallel. Q.E.D.

LEMma 5.3. Let $R$ be a canonical region of $S$ in $E$ and let $S \subset R$ be a solution curve of $S$. If $S$ is a closed orbit, $R$ is an annular region. If $S$ is a line homeomorph, then $R-S$ consists of either one or two components each of which is simply-connected.

Proof. Since a closed orbit which is not embedded in an annular neighbor- 
hood is a separatrix, every canonical region is filled either entirely by closed orbits or entirely by line homeomorphs. It is easily seen that any plane region filled entirely by closed orbits is an annular region.

Next let $R$ be filled by line homeomorphs. Clearly $R-S$ has at most two components, one lying on each side of $S$. Suppose there are two components, $A$ and $B$. There is a strip neighborhood $N_{S}$ of $S$ such that solutions in $A \cap N_{S}$ and those in $B \cap N_{S}$ are separated in $N_{S}$ by $S$.

Each solution $S^{\prime} \subset A$ has a strip neighborhood $N_{S^{\prime}}$ whose boundary in $R$ is formed by two solutions of $S$ in $R$, since the set $S_{+} \cup S_{-}$consists of separatrices and is not in $R$. Thus if $\Gamma^{\prime}$ is an open transversal for some subregion of $A$ and if one end of $\Gamma^{\prime}$ lies outside $N_{S^{\prime}}$, then $\Gamma^{\prime}$ intersects solutions lying on both sides of $S^{\prime}$ in $N_{S^{\prime}}$ if and only if $\Gamma^{\prime}$ intersects $S^{\prime}$.

Let $P$ be a point of $A$. If $P \in N_{S}$, then there is an open transversal for a subregion of $A$ through $P$, which approaches $S$ at one end. If $P \in A-N_{S}$, then join $S$ to $P$ by a curve $\pi(t), 0 \leqq t \leqq 1$, lying in $A$ except for the end point $\pi(0) \in S$, and such that $\pi(t)$ is a transversal for $A \cap N_{s}$. Let $t_{0}$ be the least upper bound of parameter values on $\pi(t)$ such that there is an open arc $\Gamma_{0}$, with one end approaching $S$, which passes through $\pi\left(t_{0}\right)$ and which is a transversal for some subregion of $A$. But then, in the strip $N_{S_{0}}$, where $\pi\left(t_{0}\right)$ $\in S_{0}, \Gamma_{0}$ does not cross $S_{0}$ and thus $\Gamma_{0}$ intersects only solutions on one side of $S_{0}$ in $N_{S_{0}}$. Therefore such an arc $\Gamma_{0}$ can always be extended locally and, since $\pi(t)$ is compact, $t_{0}=1$. Thus there is a strip parallel subregion of $A$ (filled out by solutions which intersect the extended transversal through $P$ ) which contains $A \cap N_{S}$ and $P \in A$.

Let $J$ be any simple closed Jordan curve in $A$. Since $J$ is compact, it is covered by a finite number of the parallel strip neighborhoods $N_{S^{\prime}}$. Thus there is one open $\operatorname{arc} \Gamma$ which is a transversal for a subregion of $A$ containing $A \cap N_{S}$ and also all the strips $N_{S^{\prime}}$ covering $J$. Thus $J$ is contained in a strip region, which is a simply-connected subregion of $A$, and $J$ is homotopic to a point in $A$. Therefore $A$, and similarly $B$, is simply-connected.

Next suppose $R-S$ consists of just one component. We again consider open transversals, for subregions of $R-S$, which have one end approaching $S$ and by the same argument as above, we conclude that $R-S$ is simplyconnected. Q.E.D.

THEOREM 5.2. Let $R$ be a canonical region of a differential system $S$ filling the plane $E$. Then $S$ in $R$ is a parallel region.

Proof. If $R$ contains one closed orbit, then $R$ is annular. Then assume that $R$ is filled by line homeomorphs such as $S$. If $R-S$ has two components $A$ and $B$, then construct open transversals $\Gamma_{A}$ and $\Gamma_{B}$ for $A$ and $B$ respectively. One can modify the ends of $\Gamma_{A}$ and $\Gamma_{B}$ in the parallel strip $N_{S}$ so as to join them to form one open transversal for $\mathcal{S}$ in $R=A \cup B \cup S$. Thus $S$ in $R$ is a strip region. 
If $R-S$ consists of one simply-connected component, then let $\Gamma$ be an open transversal for $R-S$. Within the strip $N_{S}$ we modify the ends of $\Gamma$ to form a simple closed Jordan curve which intersects each solution of $S$ in $R$ in exactly one point. By using a map, analogous to the one for an open transversal, we effect an $o$-equivalence between $S$ in $R$ and the punctured plane filled by $\dot{x}=x, \dot{y}=y$, Thus $S$ in $R$ is radial parallel. Q.E.D.

Corollary 5.1. Two solutions $S_{1}$ and $S_{2}$ of $S$ are parallel if and only if they are contained in the same canonical region of $\mathcal{S}$.

Proof. The proof is easy. Q.E.D.

Thus the canonical regions are parallel, homogeneous (as regards the nature of the solutions and their limit sets), intrinsic sets of decomposition for any differential system filling any plane simply-connected region. Since the canonical regions admit transversals, they admit extensions of many of the local theories of differential equations. This aspect of the significance of the canonical regions will be investigated in the final sections of this paper.

6. Combinatorial analysis of the separatrix structure of differential systems. The distinguishing of the separatrices and the decomposition of a solution family is somewhat analogous to the simplification of a topological space resulting from a triangulation. In this spirit we obtain an invariant similar to the Euler-Poincaré characteristic. For simplicity we restrict attention to the case in which the differential system $\delta$ has only one critical point.

THEOREM 6.1. Let $S$ be a differential system filling $E$ and having only a finite number $M_{S}$ of separatrices. Then the number $M_{R}$ of canonical regions is also finite. If $\mathcal{S}$ is noncritical, then $M_{R}-M_{S}=+1$. If $S$ has only one critical point $P$, and $P$ is not a limit separatrix, then $M_{R}-M_{S}=0$. If $\mathcal{S}$ has only the one critical point $P$ and if there is an unbounded separatrix which approaches $P$, then $M_{R}-M_{S}=-1$.

Proof. If $M_{R}$ were infinite, then there would be one separatrix $S$ which contained boundary points of infinitely many canonical regions $\left\{R_{1}, R_{2}, R_{3} \ldots\right\}$. But then each point of $S$ would be a boundary point for each of the canonical regions $\left\{R_{i}\right\}$ and $S$ must be a critical point. But this is impossible since at most $M_{S}$ separatrices approach $S=P$. Therefore $M_{R}$ is finite. Furthermore if $\mathcal{S}$ were noncritical, then clearly $M_{R} \leqq 2 M_{S}$.

First assume $S$ is noncritical. If we exclude one canonical region $R_{\mathbf{1}}$, we can establish a one-to-one correspondence between each remaining canonical region $R$ and the separatrix which bounds $R$ on the side nearest $R_{1}$. Thus $M_{R}-M_{S}=+1$.

Next let $P$ be the unique critical point of $S$ and assume $P$ is not a limit separatrix. Then $P$ forms one component of the boundary of an annular or spiral canonical region $R_{P}$. If $R_{P}$ is bounded, then its other boundary is a closed orbit separatrix $S_{1}$ which is not the limit of separatrices lying between 
$P$ and $S_{1}$. In fact, since $M_{S}$ is finite, there are easily seen to be no limit separatrices of $S$. Moreover we can establish a one-to-one correspondence between each canonical region and the separatrix which bounds it on the side nearest $P$ (of course, $R_{P}$ corresponds to $P$ ). Thus $M_{R}-M_{S}=0$.

Finally let the unique critical point $P$ of $S$ be a boundary point for an unbounded separatrix $S^{\prime}$ of $\delta$. We correspond each canonical region, for which $P$ is not a boundary point, with the separatrix which bounds it on the side nearest $P$. We correspond each bounded canonical region with the separatrix which bounds it away from infinity. There are just as many unbounded canonical regions which approach $P$ as there are unbounded separatrices which approach $P$. The correspondence considered has omitted $P$ itself. Thus $M_{R}-M_{S}=-1$ in this case. Q.E.D.

\section{Equivalence of differential systems}

7. Separatrix configurations as complete invariants. If two solution families $S_{1}$ and $S_{2}$ filling $E$ are $o$-equivalent, then their subfamilies $\widetilde{S}_{1}$ and $\widetilde{S}_{2}$ of separatrices are also $o$-equivalent; however the converse is false (see $\S 3$, case 7 above).

Definition. For each solution family $S$ filling $E$ define a separatrix configuration $S \subseteq$ of $\mathcal{S}$ to be a collection of curves consisting of separatrices of $\delta$ plus one representative solution chosen from each canonical region.

LEMMA 7.1. Let $S S_{1}$ and $S S_{2}$ be two separatrix configurations of a differential system $\delta$ having no limit separatrices other than critical points in E. Then there is an o-homeomorphism $T$ of $E$ onto itself which is the identity on $\mathfrak{S}_{1}=\mathfrak{S}_{2}$, maps $S \varsigma_{1}$ onto $S \Im_{2}$, and maps each canonical region of $\mathcal{S}$ onto itself.

Proof. We define $T$ on $\widetilde{S}_{1}$ as the identity map. Within each canonical region $R$, by sliding solutions along a transversal $\Gamma_{R}$, we define an $o$-homeomorphism $T_{R}^{\prime}$ of $R$ onto itself which carries the curve $S_{1} \in S S_{1}$ in $R$ onto $S_{2} \in S \Im_{2}$. In fact $T_{R}^{\prime}$ is chosen to be the identity on all solutions of $S$ in $R$ except for those intersecting $\Gamma_{R}$ in a neighborhood $N$ of the segment between $S_{1}$ and $S_{2}$ on $\Gamma_{R}$.

We shall next modify $T_{R}^{\prime}$ so that it is extended by $T$ to the boundary of $R$. If $R$ is an annular canonical region, then no modification is required. If $R$ is a strip region, then we need modify $T_{R}^{\prime}$ only near that part of the boundary of $R$ formed by $S_{1+} \cup S_{1-}$ which consists of either one or two points. By use of a technique like that of the isolating curves of Kaplan [8, p. 31], we can maintain the property that points on the solutions of $S$ which intersect $\Gamma_{R}$ in $N$ and which are near a point of $S_{1+} \cup S_{1-}$ remain near this point when $T_{R}^{\prime}$ is modified. In other words, we define an $o$-homeomorphism $T_{R}$ of the closed region $\bar{R}$ onto itself so that $T_{R}=T$ on the boundary of $R$.

Finally suppose $R$ is a spiral region. Here each of the two components of the boundary $S_{+} \cup S_{-}$consists either of one point, or a simple closed curve 
formed by a finite number of critical points and separatrices, or a curve tending to infinity along both ends and formed by a countable number of critical points and separatrices. In each of these cases the method of isolating curves enables one to modify $T_{R}^{\prime}$ to $T_{R}$, an $o$-homeomorphism of the closed region $\bar{R}$ onto itself such that $T_{R}=T$ on $\bar{R}-R$.

We designate the map, which is $T_{R}$ on each canonical region $R$, by $T$ and we show that $T$ is a homeomorphism of $E$ onto itself. Since $T$ is a one-toone transformation of $E$ onto $E$, we need only show that $T$ is continuous.

Let $P_{n} \rightarrow P$ be a sequence of points in $E$ and $T\left(P_{n}\right), T(P)$ their images. If $P$ lies within a canonical region then clearly $T\left(P_{n}\right) \rightarrow T(P)$. Suppose $P$ lies on a separatrix of $\mathcal{S}$ but all the $\left\{P_{n}\right\}$ lie in one closed canonical region $\bar{R}$. Again since $T$ is a homeomorphism on $\bar{R}, T\left(P_{n}\right) \rightarrow T(P)$. Similarly if the $P_{n}$ lie in only a finite number of closed canonical regions, then $T\left(P_{n}\right) \rightarrow T(P)$.

However if the $P_{n}$ lie in an infinite number of distinct canonical regions $\left\{\bar{R}_{n}\right\}$, then $P$ must be a critical point which is a boundary point for infinitely many of the regions $\left\{\bar{R}_{n}\right\}$. But the regions $\left\{R_{n}\right\}$ approach $P=T(P)$ uniformly, that is, all but a finite number of the $\left\{R_{n}\right\}$ lie within each neighborhood of $P$. Since $T$ maps each $R_{n}$ onto itself, almost all $T\left(P_{n}\right)$ lie within each neighborhood of $T(P)$ and $T\left(P_{n}\right) \rightarrow T(P)$. Thus $T$ is continuous and therefore an $o$-homeomorphism of $S$ in $E$ onto $S$ in $E$. Q.E.D.

THEOREM $\left.{ }^{(}\right)$7.1. Let $S_{1}$ and $S_{2}$ be differential systems filling $E$ and having no limit separatrices other than critical points. A necessary and sufficient condition that $S_{1}$ and $S_{2}$ be o-equivalent is that their separatrix configurations $S S_{1}$ and $S S_{2}$, respectively, are o-equivalent under a map $T$ of $E$ onto itself which carries $\mathfrak{S}_{1}$ onto $\mathfrak{S}_{2}$.

Proof. If $S_{1}$ and $S_{2}$ are $o$-equivalent under a map $T$, then $T$ carries $\Im_{1}$ onto $\widetilde{S}_{2}$ and $S \Im_{1}$ onto some separatrix configuration of $S_{2}$, which by the lemma can be taken to be $S S_{2}$.

Conversely assume $S \Im_{1}$ and $S \Im_{2}$ are $o$-equivalent under $T^{\prime}$ which carries $\mathfrak{S}_{1}$ onto $\mathfrak{S}_{2}$. Within each canonical region of $\boldsymbol{S}_{1}$ we shall modify $T^{\prime}$ so that it carries $S_{1}$ onto $S_{2}$. Let $R_{1}$ be a canonical region of $S_{1}$ containing the representative solution $S_{1}$ and having a transversal $\Gamma_{1}$; and let $R_{2}, S_{2}$, and $\Gamma_{2}$ be the corresponding quantities in $\delta_{2}$. Now $R_{1}$ is strip, annular, or spiral just as is $R_{2}$.

For strip or annular regions choose the direction of parametrization along $\Gamma_{1}$ and $\Gamma_{2}$ so that corresponding bounding separatrices of $R_{1}$ and $R_{2}$ are near solutions intersecting corresponding ends of $\Gamma_{1}$ and $\Gamma_{2}$. If $R_{1}$ and $R_{2}$ are spiral regions, choose $\Gamma_{1}$ and $\Gamma_{2}$ to be similarly oriented simple closed curves which intersect each solution of $S_{1}$ in $R_{1}$ or of $S_{2}$ in $R_{2}$, respectively, in exactly one point.

(6) The author wishes to thank the referee for pointing out the need for the hypothesis of "no limit separatrices", which was lacking in the original manuscript. 
If we make correspond the solutions of $S_{1}$ in $R_{1}$ and $S_{2}$ in $R_{2}$ having the same intercepts on $\Gamma_{1}$ and $\Gamma_{2}$, respectively, we can easily define an $o$-homeomorphism of $\delta_{1}$ in $R_{1}$ onto $\delta_{2}$ in $R_{2}$. Using the techniques of Kaplan's isolating curves in a manner indicated in the lemma, we can construct $T_{R}$, an $o$-homeomorphism of $S_{1}$ in $R_{1}$ onto $S_{2}$ in $R_{2}$ such that $T_{R}$ is extended by $T^{\prime}$ to map $\bar{R}_{1}$ onto $\bar{R}_{2}$. Thus we can define a modified one-to-one map $T$ of $E$ onto $E$ such that $T=T^{\prime}$ on $\varsigma_{1}, T$ carries solutions of $S_{1}$ onto those of $S_{2}$ and $T^{-1}$ carries solutions of $\delta_{2}$ onto those of $S_{1}$, and $T$ is an $o$-homeomorphism of each closed canonical region of $S_{1}$ onto the corresponding closed canonical region of $S_{2}$.

Since $T$ is evidently one-to-one, we need only show that it is continuous. However, just as in the lemma, the only limit separatrices are critical points, and if a critical point $P$ is in the boundary of an infinite number of canonical regions, then these canonical regions approach $P$ uniformly. Thus, using the proof of the lemma, $P_{n} \rightarrow P$ implies $T\left(P_{n}\right) \rightarrow T(P)$ if $P$ is either within a canonical region of $S_{1}$, on a nonlimit separatrix of $S_{1}$, or at a critical point of $S_{1}$. Therefore $T$ is continuous. Q.E.D.

This theorem distinguishes the separatrix configurations as complete invariants for the topological theory of differential systems. The advantage of reducing the topological study from all solutions of $S$ to only those of $S \subseteq$ is that the latter is often finite or countable. Thus the nature of $S \subseteq$ is often such that one can tell at a glance if two differential systems are $o$-equivalent.

Corollary 7.1. Let $S_{1}$ and $S_{2}$ be differential systems filling $E$ and having no limit separatrices other than critical points. $A$ necessary and sufficient condition that $S_{1}$ is topologically equivalent to $S_{2}$ (under any homeomorphism of $E$ onto $E$ ) is that $S \subseteq_{1}$ and $S S_{2}$ are equivalent under a homeomorphism $T$ which maps $\mathfrak{S}_{1}$ onto $\mathfrak{S}_{2}$.

The above theorem and its corollary form a justification for the sketches, customary in nonlinear mechanics, of a few solutions which are to indicate an entire solution family. One notes that these sketches almost always are actually a separatrix configuration which does determine a unique (in a qualitative sense) differential system.

One can introduce a useful partial ordering into the collection of all $o$-equivalent classes of differential systems filling the plane by stating that the simplest differential systems are those having the simplest (for example, the smallest cardinal) separatrix configurations.

8. Equivalence of noncritical differential systems. We next consider the special case in which $S$ has no critical points in $E$ and we obtain an extension of a result of Kaplan [8, p. 43]. Here every triple $S_{1}, S_{2}, S_{3}$ of distinct solutions of $S$ satisfy one of five positional relations: $S_{1}\left|S_{2}\right| S_{3}$ (read $S_{2}$ separates $S_{1}$ and $\left.S_{3}\right), S_{1}\left|S_{3}\right| S_{2}, S_{2}\left|S_{1}\right| S_{3},\left|S_{1} S_{2} S_{3}\right|+\left(\right.$ read $S_{1}, S_{2}, S_{3}$ in this order form a counterclockwise cyclic triple), and $\left|S_{1} S_{3} S_{2}\right|+\equiv\left|S_{1} S_{2} S_{3}\right|-$. The solu- 
tions of $\delta$ together with these relations satisfy certain natural axioms $[8$, p. 165] and are thus a realization of an abstract chordal system. The study of chordal systems enables us to replace the topological hypotheses of the preceding theorem by algebraic conditions.

It is clear that whatever representative solution is chosen from each canonical region of $\mathcal{S}$ in defining $S \subseteq$, the resulting chordal system $S \subseteq$ is determined up to isomorphism $\left({ }^{7}\right)$; moreover the isomorphism can be taken as the identity on the chordal subsystem $\mathfrak{S}$.

Theorem 8.1. Let $S_{1}$ and $S_{2}$ be noncritical differential systems filling $E$. A necessary and sufficient condition that $S_{1}$ and $S_{2}$ be o-equivalent is that the chordal systems $S \widetilde{S}_{1}$ and $S_{2}$ are isomorphic under a correspondence $T$ which carries $\mathfrak{\Im}_{1}$ onto $\mathfrak{\Im}_{2}$.

Proof. The necessity is immediate. To prove the sufficiency one needs only to extend $T$ to an isomorphism of the chordal system of all solutions of $S_{1}$ onto that of all solutions of $S_{2}$, see [8, p. 43].

Since the separatrices bounding one canonical region of $\delta_{1}$ are distinguished positionally (see following definition for details), the boundary of each canonical region of $S_{1}$ is mapped by $T$ onto the boundary of one canonical region of $S_{2}$. Furthermore the representative curves of $S \Im_{1}-\widetilde{S}_{1}$ are mapped by $T$ onto the representative curves of $S \Im_{2}-\Im_{2}$ lying in the corresponding canonical regions. Also corresponding curves of $S \Im_{1}-\Im_{1}$ and of $S \Im_{2}-\Im_{2}$ are similarly related to the corresponding bounding separatrices because $T$ is an isomorphism of $S \Im_{1}$ onto $S \Im_{2}$.

Each canonical region is strip parallel and by relating one representative curve of a canonical region to the bounding separatrices, one prescribes the relations of every solution curve within the canonical region to the bounding separatrices. Using transversals within corresponding canonical regions of $S_{1}$ and $S_{2}$, we define the map $T$ from all solutions of $S_{1}$ onto all solutions of $S_{2}$ so that $T$ is an isomorphism on the chordal subsystems of solutions of $S_{1}$ onto the solutions of $S_{2}$ which lie within each corresponding pair of closed canonical regions.

Since positional relations between solutions of different canonical regions are determined by the chordal systems $\varsigma_{1}$ and $\varsigma_{2}$ in $\delta_{1}$ and $S_{2}$ respectively, $T$ defines an isomorphism of $\delta_{1}$ onto $\delta_{2}$. Therefore, by Kaplan's theorem, $\delta_{1}$ is $o$-equivalent to $S_{2}$. Q.E.D.

Corollary 8.1. Let $S_{1}$ and $S_{2}$ be noncritical differential systems filling $E$. $A$ necessary and sufficient condition that $S_{1}$ be topologically equivalent to $S_{2}$ (under any homeomorphism of $E$ onto $E$ ) is that the chordal systems $S \widetilde{S}_{1}$ and $S \widetilde{S}_{2}$

( ${ }^{7}$ An isomorphism between two chordal systems is a one-to-one transformation which preserves the relations of separation and positive cyclicness. An anti-isomorphism is a one-toone transformation which preserves the relation of separation but which maps positive cyclic sets into negative cyclic sets. 
are either isomorphic or anti-isomorphic under a correspondence $T$ which maps $\mathfrak{\Im}_{1}$ onto $\mathfrak{\Im}_{2}$.

The total number of canonical regions of a differential system $S$ is at most countable. If $S$ is noncritical, each canonical region is bounded by a finite or countable cyclic set of separatrices. Thus for noncritical differential systems $\mathcal{S}$ which contain no limit separatrices, $S \mathfrak{S}$ is always finite or countable. This presents an advantage over the corresponding theorem of Kaplan which considers the noncountable chordal system $\mathcal{S}$.

9. Existence of topological classes of noncritical differential systems. The preceding two sections established uniqueness theorems for topological classes of differential systems having prescribed separatrix configurations. We now turn to the existence problem for a differential equation realizing a prescribed separatrix configuration. We restrict our attention here to noncritical differential systems filling the plane.

Definition. Let $\Sigma=\left\{S_{\alpha}\right\}$ be an abstract chordal system. A subset $\sigma \subset \Sigma$ is said to be (or to bound) a canonical region of $\Sigma$ in case either (1) $\sigma$ consists of one element which divides $\Sigma$ into two sets, exactly one of which is empty, or (2) $\sigma$ is a cyclic subset of two or more elements of $\Sigma$ such that no element of $\Sigma-\sigma$ separates two members of $\sigma$, and $\sigma$ is not properly contained in any subset of $\Sigma$ having this property.

Definition. A chordal supersystem $S \Sigma$ of a chordal system is a (proper) extension of $\Sigma$ in case the elements of $S \Sigma-\Sigma$ correspond one-to-one with the canonical regions of $\Sigma$ and furthermore (1) if the element $S_{\sigma} \in S \Sigma-\Sigma$ corresponding to the canonical region $\sigma \subset \Sigma$ is adjoined to $\Sigma$, then $\sigma$ is no longer a canonical region, and (2) each pair of elements of $S \Sigma-\Sigma$ contain between them at least two elements of $\Sigma$.

For noncritical differential systems $\mathcal{S}, S \subseteq$ is an extension of the chordal system $\mathfrak{S}$. Also the canonical regions of the chordal system $\mathfrak{S}$ are precisely the sets of separatrices which bound each canonical region of the solution family $S$.

THEOREM 9.1. Let $\Sigma$ be an abstract chordal system admitting an extension S氵. Assume

(1) $\Sigma$ is countable,

(2) each element of $\Sigma$ belongs to exactly two canonical regions of $\Sigma$,

(3) each pair of elements $S_{1}$ and $S_{2}$ of $\Sigma$ can be joined by a finite chain $K_{1}, K_{2}, \cdots, K_{n}$ of canonical regions of $\Sigma$ such that $K_{i}$ and $K_{i+1}, i=1,2, \cdots$, $n-1$, are adjacent (have one common element) and $S_{1} \in K_{1}, S_{2} \in K_{n}$.

Then there exists a unique (up to o-equivalence) noncritical differential system $S$ filling $E$ such that there is an isomorphism $T$ of $S \Sigma$ onto $S \Im$ which maps $\Sigma$ onto $\mathfrak{S}$. Also $\mathcal{S}$ has no limit separatrices and every noncritical differential system having no limit separatrices is the realization of an abstract pair $(\Sigma, S \Sigma)$ satisfying the hypotheses. 
Proof. Select a canonical region $\sigma_{1}$ of $\Sigma$ and inscribe in the plane open unit disc a cyclic set of chords $T \sigma_{1}$ isomorphic to the chordal subsystem $\sigma_{1}$. Such a cyclic set of chords $T \sigma_{1}$ is finite or else countable and the chords approach the circumference uniformly.

Fill the interior region of $T \sigma_{1}$ with a strip parallel regular curve family $\delta \sigma_{1}$ whose curves bear the same positional relations to the boundary chords as does the element of $S \Sigma-\Sigma$ lying in $\sigma_{1}$ bear to $\sigma_{1}$. Moreover construct $S \sigma_{1}$ so that each chord of $T \sigma_{1}$ has a half-neighborhood in which the curves of $S \sigma_{1}$ consist of straight line segments parallel to the boundary chord.

Note that those elements $\sigma_{2} \subset \sigma_{1}$, such that the $T \sigma_{2}$ all intersect the concentric circle of radius $1 / 2$, are finite in number and each of them is part of the boundary of a canonical region adjacent to $\sigma_{1}$. External to each of the chords $T \sigma_{2}$ we inscribe chords in the unit disc corresponding to the canonical regions of $\Sigma$ which are adjacent to $\sigma_{1}$ and which contain the elements of $\sigma_{2}$.

Continue in this manner; in the $n$th stage note the subset of all the chords which have been hitherto inscribed in the unit disc and which intersect the concentric circle of radius $(n-1) / n$. The corresponding finite set of elements of $\Sigma$ each bounds a canonical region of $\Sigma$ which is adjacent to one already mapped into the disc and which is now mapped into the exterior region of the proper chord in the disc. As prescribed by $S \Sigma-\Sigma$ we fill the interiors of each of these new cyclic sets of chords by a strip parallel regular curve family which consists of parallel straight line segments near the boundary chords.

Because of the finite chain condition each element of $\Sigma$ will be mapped by $T$ into a chord of the unit disc after a finite number of stages. Also since the chords inscribed in the $n$th stage are each exterior to the plane region filled at the $(n-1)$ th stage, we have defined a process for filling a simplyconnected subregion of the unit disc by a regular curve family $S^{\prime}$, which is the union of all the $\delta_{\sigma}$ where $\sigma$ is a canonical region of $\Sigma$.

The only possible separatrices of $S^{\prime}$ are the chords $T \Sigma$. Let $S \in \Sigma$ belong to the two adjacent canonical regions $\sigma^{\prime}$ and $\sigma^{\prime \prime}$ of $\Sigma$. Let $N$ be a neighborhood in the disc of $T S$ and let $N$ be bounded by exactly the two curves $T S^{\prime}$ and $T S^{\prime \prime}$ of $S^{\prime}$ lying interior to $T \sigma^{\prime}$ and $T \sigma^{\prime \prime}$ respectively. Since each pair of elements of $S \Sigma-\Sigma$ contain between them at least two elements of $\Sigma$, there are two elements $T S$ and $T \tilde{S}$ of $T \Sigma$ in $N$. But if $N$ is strip parallel, then the region between $T S$ and $T \tilde{S}$ contains neither $T S^{\prime}$ nor $T S^{\prime \prime}$, contradicting the assumption that $\sigma^{\prime}$ and $\sigma^{\prime \prime}$ are adjacent to $S$. Thus $T S$ is a separatrix of $S^{\prime}$.

The map $T$ can therefore be defined as an isomorphism of $S \Sigma$ onto a separatrix configuration of $S^{\prime}$ so that $\Sigma$ is carried onto the separatrices of $S^{\prime}$. Also $S^{\prime}$ contains no limit separatrices since each separatrix of $S^{\prime}$ belongs to the boundary of two canonical regions of $S^{\prime}$.

By Kaplan's theorem [10, p. 514], $S^{\prime}$ is $o$-equivalent to an exact, analytic, noncritical differential system $S$ filling $E$. The chordal system pair $(\mathfrak{S}, S \subseteq)$ of $\mathcal{S}$ is unique, as is $\mathcal{S}$ itself, up to $o$-equivalence, by Theorem 8.1. 
Every noncritical differential system having no limit separatrices in $E$ has a separatrix configuration satisfying the hypotheses of this theorem and can therefore be constructed from an abstract pair $(\Sigma, S \Sigma)$ as above. Q.E.D.

Thus the question of existence of classes of noncritical differential systems having no limit separatrices is reduced to the purely algebraic problem of considering abstract chordal systems $\Sigma$ with their extensions $S \Sigma$. A brief investigation reveals the following cases of interest.

REMARK 1. By Theorem 5.1 there is a unique (up to $o$-equivalence) differential system in $E$ with no separatrices.

REMARK 2. If $\Sigma$ contains just one element, then $\Sigma$ admits no proper extensions and thus there is no noncritical differential system in $E$ with exactly one separatrix.

REMARK 3. If $\Sigma$ has two elements, then there corresponds a unique pair $(\Sigma, S \Sigma)$ and thus there is in $E$ a unique noncritical differential system with exactly two separatrices.

REMARK 4. If $\Sigma$ contains three elements, there are four nonisomorphic pairs $(\Sigma, S \Sigma)$ and thus four distinct noncritical differential systems in $E$ each having exactly three separatrices.

REMARK 5. The cardinality of all the $o$-equivalence classes of noncritical differential systems having no limit separatrices in $E$ is the same as the cardinality of all $o$-equivalence classes of regular curve families in $E$, that is, the power of the continuum.

\section{APPLICATIONS TO GLOBAL THEORIES OF DIFFERENTIAL SYSTEMS}

\section{Construction of global integrals.}

Definition. An integral of a differential system $S$ defined in a plane region $R$ is a real-valued function $\psi(x, y) \in C^{(1)}$ in $R$, constant along each solution curve of $S$, but constant on no open subset of $R$.

Kamke [7, p. 56] has constructed global integrals for noncritical differential systems in bounded subregions of $E$ and Digel [4, p. 231] for differential systems comprised of closed solution curves about a unique critical point. Kaplan [8, p. 183] has constructed "continuous integrals" for every regular curve family filling $E$. However Ważewski [19, p. 104] has constructed a noncritical differential system of class $C^{(\infty)}$ which does not admit a (class $\left.C^{(1)}\right)$ integral in $E$. We shall extend the results of Kamke and Digel and distinguish an important class of differential systems which do admit global integrals in the plane $E$.

If a differential system $S$ contains either a spiral canonical region or a critical point $P$ which is a limit point for a nonseparatrix solution of $S$, then it is clear that $S$ cannot admit even a "continuous integral." Thus we shall restrict our attention to those differential systems which do not have such structures. 
Of course, if $\delta$ contains no separatrices, then $\delta$ admits a nonsingular, analytic, transversal which is nowhere tangent to $\delta$, see $[15$, p. 320]. In this case $S$ is $o$-equivalent to $y^{\prime}=0$ in $E$, and this even under a $C^{(1)}$-homeomorphism, so that $\delta$ admits an integral in $E$, see [1, p. 9].

THEOREM 10.1. Let $S$ be a differential system filling $E$ and containing no spiral canonical regions and no limit separatrices. Then there exists a global integral $\psi(x, y) \in C^{(1)}$ of $\mathcal{S}$ in $E$ such that:

(1) the real variety $\psi_{x}^{2}+\psi_{y}^{2}=0$ is nowhere dense and has plane Lebesgue measure zero,

(2) $0 \leqq \psi(x, y) \leqq 1$,

(3) each component of the real variety $\psi(x, y)=C>0$ is one entire nonseparatrix solution of $\mathcal{S}$ and conversely for every nonseparatrix solution $S$ of $\mathcal{S}$ there is a positive constant $C$ such that $S$ is one component of the real variety $\psi(x, y)=C$,

(4) the real variety $\psi(x, y)=0$ is precisely $\subseteq$, the union of all separatrices of S.

Proof. A critical point bounds only one (annular) canonical region but every other type of separatrix bounds exactly two canonical regions. Thus $S \subseteq$ is nowhere dense in $E$ and, being countable, $S \subseteq$ has plane Lebesgue measure zero.

Let $R$ be a canonical region of $S$ with open transversal $\Gamma(t), 0<t<1$, which is a nonsingular, analytic arc nowhere tangent to a solution of $S$. Each component of the boundary of $R$ in $E$ consists of either one critical point or of one line homeomorph separatrix which tends to infinity in both directions.

We prescribe an initial real-valued function $\phi(t), 0<t<1$, on $\Gamma$ such that $0<\phi(t) \leqq 1, \phi(t) \in C^{(1)}, \dot{\phi}=0$ only at $t=.5, \lim _{t=0} \phi(t)=\lim _{t=1} \phi(t)=0$. Define an integral function by $\psi_{R}(x, y)=\phi(t)$ where $S_{t}$ is the solution through $(x, y)$ and intersecting $\Gamma$ at the parameter value of $t$. Then $\psi_{R}(x, y) \in C^{(1)}$ in $R$ and $\operatorname{grad} \psi_{R}=0$ only on $S_{\text {.5 }}$. Furthermore the function $\phi(t)$ can be chosen so as to approach zero so rapidly at $t=0$ and $t=1$ that, if we define $\psi_{R}(x, y)=0$ for $(x, y)$ on each of the countably many components of $\bar{R}-R$, then $\psi_{R}(x, y)$ $\in C^{(1)}$ in $\bar{R}$ and also grad $\psi_{R}=0$ on $\bar{R}-R$.

In each canonical region $R_{\alpha}$ of $S$ we similarly define an integral function $\psi_{R_{\alpha}}(x, y)$ and then let $\psi(x, y)=\psi_{R_{\alpha}}(x, y)$ for $(x, y) \in \bar{R}_{\alpha}$. Then $0 \leqq \psi(x, y) \leqq 1$ is in class $C^{(1)}$ in $E$ and is an integral for $\delta$. Also the real variety grad $\psi=0$ consists of a separatrix configuration $S \subseteq$ which is nowhere dense and has plane Lebesgue measure zero. The real variety $\psi(x, y)=0$ consists of the separatrices $\subseteq$ of $\mathcal{S}$ and for each positive constant $C$, the real variety $\psi(x, y)$ $=C$ consists of at most two solutions from each canonical region of S. Q.E.D.

Corollary 10.1. If $S$ is of class $C^{(\alpha)}, \alpha=1,2,3, \cdots, \infty, \omega$ (analytic) with no limit separatrices and no spiral canonical regions in $E$, then the global integral $\psi(x, y)$ defined in the theorem is of class $C^{(\alpha)}$ within each canonical region. 
Corollary 10.2. Let $\delta$ be in class $C^{(\alpha)}, \alpha=2,3, \cdots, \infty, \omega$ with no limit separatrices and no spiral canonical regions in $E$. Define the invariant $\mu$-measure on Lebesgue measurable sets $\{\chi\}$ by the Lebesgue integral $m_{\mu}(\chi)=\iint_{\chi} \mu(x, y) d x d y$ where $\mu(x, y)=\left[\left(\psi_{x}^{2}+\psi_{y}^{2}\right) /\left(f^{2}+g^{2}\right)\right]^{1 / 2}$, except at critical points of $\mathcal{S}$ where $\mu(x, y)$ $=0$. Then the $\mu$-measure is absolutely continuous with respect to Lebesgue measure and vice versa, see [17].

Of the eleven cases of differential systems discussed in $\S 4$, the first nine do admit global integrals in $E$ as stated in Theorem 10.1. An open question is, "Does every noncritical analytic differential system possess a global integral in the plane?" Kaplan [10, p. 514] has shown that there is a noncritical, exact, analytic differential system in each $o$-equivalence class of regular curve families filling the plane. Thus this question seems difficult from the topological viewpoint.

For each existence theorem for an integral of an ordinary differential system, there corresponds an existence theorem for a principal integral of a first order, homogeneous, linear partial differential equation. Even for some nonhomogeneous, linear, partial differential equations integration along the characteristic curves enables one to construct integrals or solutions in the large.

THEOREM 10.2. Let $P: f(x, y) Z_{x}+g(x, y) Z_{y}=h(x, y) k(Z)$ have a characteristic differential system $S$ of class $C^{(\alpha)}, \alpha=1,2, \cdots, \infty, \omega$ having no separatrices in the plane $E$. Let $h(x, y) \in C^{(\alpha)}$ in $E$ and $k(Z) \in C^{(\alpha-1)}$ for some interval $I: a<Z<b$. Suppose for some real number $Z_{0} \in I$ the function $K(Z)$ $=\int_{Z_{0}}^{Z} d u / k(u)$ is defined for all $Z \in I$ and the map $K(Z) \rightarrow Z$ is a $C^{(\alpha)}$-homeomorphism of the real line onto $I$. Then there exists an integral $\psi(x, y)$ of $\mathcal{P}$ in $E$ such that:

(1) $\psi(x, y) \in C^{(\alpha)}$ in $E$,

(2) $a<\psi(x, y)<b$,

(3) $f \psi_{x}+g \psi_{y}=h k(\psi)$ identically in $(x, y)$.

Proof. The proof is easy, see [15, p. 326]. Q.E.D.

Corollary 10.3. Let $S: \dot{x}=f(x, y), \dot{y}=g(x, y)$ be a differential system of class $C^{(\alpha)}, \alpha=2,3, \cdots, \infty, \omega$ in the plane $E$ where $S$ has no separatrices. Then there exists a "density function" $\mu(x, y) \in C^{(\alpha-1)}$ in $E$ which satisfies the "equation of continuity" for a steady state compressible fuid; that is,

(1) $(\mu f)_{x}+(\mu g)_{y}=0$,

(2) $\mu(x, y)>0$.

Proof. The equation $f \mu_{x}+g \mu_{y}=-\left(f_{x}+g_{y}\right) \mu$ is of the type considered in the theorem with $k(\mu)=\mu$ and $I: 0<\mu<\infty$. Q.E.D.

11. Invariants of entire transformation groups. We shall study only the invariants of differential systems $S: \dot{x}=f(x, y), \dot{y}=g(x, y)$ under one-parameter Lie groups of transformations whose infinitesimal groups are differential 
systems $U: \dot{x}=\xi(x, y), \dot{y}=\eta(x, y)$ in $E$, each trajectory of which is defined for all $-\infty<t<\infty$, see [16]. As in the usual Lie theory [13, p. 105], $S$ is invariant under the group (for each $t$, the trajectories of $V$ define an $o$-equivalence of $S$ in $E$ onto itself) if and only if there exists a real function $\lambda(x, y)$, vanishing on the critical points of $S$ and continuous elsewhere, and such that $[S, V]=\lambda(x, y) S$, that is

$$
\xi f_{x}+\eta f_{y}-f \xi_{x}-g \xi_{y}=\lambda f \text { and } \xi g_{x}+\eta g_{y}-f \eta_{x}-g \eta_{y}=\lambda g .
$$

REMARK. If $\xi g-\eta f=0$ at one point of a solution $S \in \mathcal{S}$, then $\xi g-\eta f=0$ everywhere on $S$ and each transformation generated by $U$ maps $S$ onto itself. In particular the critical points of $S$ are individually invariant under $U$.

THEOREM 11.1. Let the infinitesimal group $V: \dot{x}=\xi(x, y), \dot{y}=\eta(x, y)$ leave the differential system $S: \dot{x}=f(x, y), \dot{y}=g(x, y)$ invariant in the plane $E$. Then $\xi g-\eta f=0$ on each separatrix $S \in \mathcal{S}$ and $S$ is mapped onto itself by $U$.

Proof. If $\xi g-\eta f \neq 0$ on a separatrix $S \in \mathcal{S}$, then $S$ would be embedded in a parallel neighborhood $N$ filled by translates of $S$ and such that $N$ is bounded by just two translates of $S$ plus $S_{+} \cup S_{-}$. Moreover each solution of $\mathcal{S}$ in $N$ has the same limit sets. But this contradicts the hypothesis that $S$ is a separatrix. Q.E.D.

If $U$ leaves $S$ invariant in $E$ and if, within some simply-connected plane region $R, \xi g-\eta f \neq 0$, then $\mu=(\xi g-\eta f)^{-1}$ is an integrating factor for $S$ in $R$. Since the separatrices of $\mathcal{S}$ are invariant under $V$, each canonical region of $\mathcal{S}$ is also individually invariant under $U$. The next theorem demonstrates that the Lie integrating factor $\mu=(\xi g-\eta f)^{-1}$ could be of use within one canonical region of $S$, at most.

Theorem 11.2. Let the infinitesimal group $V: \dot{x}=\xi(x, y), \dot{y}=\eta(x, y)$ leave the differential system $S: \dot{x}=f(x, y), \dot{y}=g(x, y)$ invariant in $E$. If $\xi g-\eta f \neq 0$ in $E$, then neither $U$ nor $S$ has any separatrices in $E$.

Proof. Both $U$ and $\delta$ are noncritical in $E$ and, by Theorem 11.1, $\delta$ has no separatrices in $E$. Then there is a $C^{(1)}$-homeomorphism $T$ of $E$ onto itself which carries $S$ onto $T S$ consisting of the lines $x=$ constant. Also $T$ maps $V$ onto the infinitesimal group $T V$ (which may be only continuous) which leaves $T S$ invariant.

Since the trajectories of $T V$ (images of trajectories of $V$ ) are nowhere tangent to $T S$, each such trajectory lies along a curve $y=y(x) \in C^{(1)}$. Suppose one such solution curve $y=y(x)$ of $T U$ were not defined for all $-\infty<x$ $<\infty$ but, say, only for $x<0$.

Then none of the translates of $x=0$ under $T U$ lie in the left half-plane and thus $T U$ must be tangent to $T S$ on $x=0$. But this implies that $V$ is somewhere tangent to $S$ and that $\xi g-\eta f=0$ at the point of tangency. This contradicts the hypothesis. Therefore every solution of $T U$ is defined for 
all $-\infty<x<\infty$ and the solution family of $T U$ admits the $y$-axis as an open transversal. Therefore $T U$ and thus $U$ has no separatrices in E. Q.E.D.

We next mention briefly some classes of differential systems $\mathcal{S}$ invariant under certain special transformation groups $U$ in $E$.

REMARK 1. If $U$ contains no separatrices, then $\delta$ has no critical points, no limit separatrices, and every canonical region of $\delta$ is bounded by one or two separatrices.

Remark 2. Note $S: \dot{x}=f(x), \dot{y}=1$ with $f(x) \in C^{(1)}$ is invariant under $V: \dot{x}=0, \dot{y}=1$ and also $S$ has no separatrices in $E$. Similarly $\dot{x}=1, \dot{y}=f(x)$ is invariant under $\dot{x}=0, \dot{y}=1$ and has no separatrices, compare $\S 4$, case 4 .

REMARK 3. If $U$ contains a closed orbit, then $S$ contains a critical point. If $\delta$ contains a closed orbit, then $U$ contains a critical point.

REMARK 4. If $V$ is $o$-equivalent to $\dot{x}=x, \dot{y}=y$ [18], then either $S$ contains no separatrices or else $\mathcal{S}$ has a finite number of separatrices, one critical point, and a finite number of line homeomorphs joining the critical point to infinity. If there is more than one separatrix, then each canonical region of $\mathcal{S}$ is of strip type bounded by the critical point and two other separatrices. If the critical point is the only separatrix, then there is just one canonical region of $S$ and this is of annular or spiral type.

REMARK 5. If $V$ is $o$-equivalent to $\dot{x}=-y, \dot{y}=x$, then $\delta$ has a unique critical point and every other separatrix is a simple closed curve about the critical point. Each canonical region of $S$ is either annular or spiral type.

\section{BIBLIOGRAPHY}

1. I. Bendixson, Sur les courbes défines par des équations differentielles, Acta Math. vol. 24 (1901) pp. 1-88.

2. L. Berwald, Über Systeme von Gewöhnlichen Differentialgleichungen zweiter Ordnung deren Integralkurven mit dem System der geraden Linien topologisch Aequivalent sind, Ann. of Math. vol. 48 (1947) pp. 193-215.

3. E. Cartan, Leçon sur la théorie des espace à connexion projective, Paris, Gauthier, 1937, pp. 242-247.

4. E. Digel, Über die Existenz von Integralen der partiellen Differentialgleichung von $f z_{z}+g z_{y}=0$ in der Umgebung eines singulären Punkt, Math. Zeit. vol. 42 (1937) pp. 231-237.

5. G. Halphen, Sur les invariants differentielles, Thèse, Paris, 1878. 1943.

6. W. Hurewicz, Ordinary differential equations, Mimeographed notes at Brown University,

7. E. Kamke, Über die partielle Differentialgleichungen $f z_{x}+g z_{y}=h$, Math. Zeit. vol. 41 (1936) pp. 56-66; vol. 42 (1936) pp. 287-300.

8. W. Kaplan, Regular curve families filling the plane, I and II, Duke Math. J. vol. 7 (1940) pp. 154-185; vol. 8 (1941) pp. 11-46.

9. - The structure of a curve family on a surface in the neighborhood of an isolated singularity, Amer. J. Math. (1942).

10. - Topology of level curves of harmonic functions, Trans. Amer. Math. Soc. vol. 63 (1948) pp. 514-522.

11. S. Lefschetz, Lectures in differential equations, Princeton, 1946.

12. R. Liouville, Sur les invariants de certaines équations différentielles, J. Ecole Polytech. vol. 59 (1889) pp. 7-76. 
13. S. Lie and G. Scheffers, Vorlesungen über Differentialgleichungen mit bekannten Infinitesimalen Transformationen, Leipzig, 1891.

14. L. Markus, Differential equations in the large, Thesis, Harvard University, 1951.

15. - Global integrals of $f z_{x}+g z_{y}=h$, Académie Royale de Belgique, Bulletin de la Classe des Sciences (5) vol. 38 (1952) pp. 311-332.

16. - Escape times for differential equations, Rendiconti di Torino, 1952.

17. - Invariant measures defined by differential equations, Proc. Amer. Math. Soc. vol. 4 (1953) pp. 89-91.

18. G. Silov, Integral curves of a homogeneous equation of first order, Uspehi Matematiceskih Nauk N.S. vol. 5 (1950) pp. 193-203.

14. T. Ważewski, Sur l'équation $p+Q q=0$, Mathematica vol. 8 (1934) pp. 103-116.

15. H. Whitney, Regular families of curves, Ann. of Math. vol. 34 (1933) pp. 244-270.

HARVARD UNIVERSITY,

Cambridge, Mass. 